\title{
In vitro induction of mouse meningeal-derived iPS cells into neural-like cells
}

\author{
XU Jing ${ }^{1,2}$, WANG Hao ${ }^{1}$, LIANG Tao ${ }^{1,2}$, CAI XiangSheng ${ }^{1}$, RAO XiuRong ${ }^{1}$, HUANG $^{2}$ ZhenBo $^{1}$ \& \\ SHENG GuoQing ${ }^{1 *}$ \\ ${ }^{1}$ Key Laboratory of Regenerative Biology, Laboratory of Stem Cell Therapy, Guangzhou Institute of Biomedicine and Health (GIBH), Chinese \\ Academy of Sciences, Guangzhou 510530, China; \\ ${ }^{2}$ School of Life Sciences, University of Science and Technology of China, Hefei 230026, China
}

Received November 29, 2010; accepted February 18, 2011; published online April 13, 2011

Previous research has shown that mouse embryonic stem (ES) cells can be induced to form neural cells in adherent monocultures. In this study, pluripotent stem (iPS) C5 cells derived from meningeal membranes were converted successfully into neural-like cells using the same protocol generally used for ES cells. Meningeal-iPS C5 cells were induced to express neural markers Sox1, Sox3, Pax6, Nestin and Tuj1 and to reduce the expression of ES markers Oct4 and Nanog during neural differentiation, and can be differentiated into Pax6 and Nestin positive neural progenitors, and further into neuronal, astrocytic, and oligodendrocytic cells. In vitro differentiation of iPS cells into patient-specific neural cells could serve as a model to study mechanisms of genetic diseases and develop promising candidates for therapeutic applications in dysfunctional or aging neural tissues. Meningeal cells express a high level of the embryonic master regulator Sox2, allowing them to be reprogrammed into iPS cells more easily than other somatic cells.

induced pluripotent stem cells, embryonic stem cells, meningeal membranes, neural differentiation, serum-free medium, adherent monoculture

Citation: $\quad$ Xu J, Wang H, Liang T, et al. In vitro induction of mouse meningeal-derived iPS cells into neural-like cells. Chinese Sci Bull, 2011, 56: 1556-1561, doi: $10.1007 / \mathrm{s} 11434-011-4436-9$

Neurological disorders, such as Alzheimer's disease, Parkinson's disease and stroke, share a marked pathological feature, that is, the loss of a specific spectrum of cell types in different areas of the central nervous system (CNS) [1]. In most cases, the cause of these disorders is unknown [2]. The lack of effective preventative therapies and the limitations of current drug therapies, have led to efforts to develop and implement surgical interventions, such as transplantation therapies [1]. It is possible that appropriate cell types, derived from either embryonic stem (ES) cells or tissue-specific stem cells, may be used to replace cells lost during disease [3-6].

Generation of pluripotent stem (iPS) cells, sharing simi-

*Corresponding author (email: sheng_guoqing@gibh.ac.cn) lar properties to ES cells, from somatic cells was first reported by Takahashi and Yamanaka, in 2006 [7]. iPS cells derived from individual patients may be useful for drug screening or regenerative therapies. Recent publications have reported multiple technologies for iPS cell generation, including different transcription factor combinations, tissue origins and selection methods [8-19]. Although iPS cell lines generated independently in various laboratories have been shown to express pluripotency genes and to be able to differentiate into cell types of all three germ layers, it remains unclear whether iPS cells produced by these different reprogramming methods can be therapeutically useful.

Neural stem cells and meningeal cells expressing a high level of the embryonic master regulator Sox 2 can be used to generate iPS cells more easily [13,20,21]. However, iPS 
cells derived from meningeal cells are more suitable for cell transplantation therapies because of the source limitation of neural stem cells. In this work, we investigate neural differentiation from mouse meningeal-iPS C5 cells. iPS C5 cells can be induced to form neural progenitor cells, and further differentiate into neurons, astrocytes, and oligodendrocytes in a serum-free adherent monoculture system previously used for neural differentiation from mouse ES cells in our laboratory. iPS C5 cells exhibit similar features to ES cells in terms of neural differentiation in vitro.

\section{Materials and methods}

\subsection{Culture of mouse meningeal-iPS cells}

Mouse meningeal-iPS C5 cells, derived using the methods described by Qin et al. [13], were grown on mitomycin-inactivated mouse embryonic fibroblasts (MEFs) at $37^{\circ} \mathrm{C}$ and $5 \% \mathrm{CO}_{2}$ in standard ES cell medium (containing Dulbecco's Modified Eagle Medium (Gibco), 15\% fetal bovine serum (PAA), $1 \mathrm{mmol} / \mathrm{L}$ l-glutamine (Gibco), 0.1 $\mathrm{mmol} / \mathrm{L}$ nonessential amino acids (Gibco), $0.1 \mathrm{mmol} / \mathrm{L} \beta$ mercaptoethanol (Gibco), and $1000 \mathrm{U} / \mathrm{mL}$ murine leukemia inhibitory factor (LIF) (Invitrogen)). Cells were passaged every other day by incubation with $0.05 \%$ trypsin and $0.04 \%$ ethylenediaminetetraacetic acid (Gibco) for $5 \mathrm{~min}$ at $37^{\circ} \mathrm{C}$. The cultures were gently dissociated and replated at a $1: 3-1: 8$ split ratio.

\subsection{Neural differentiation from mouse meningeal-iPS cells}

For initial neural differentiation, iPS C5 cells were cultured without feeder cells on $0.1 \%$ gelatin (Sigma)-tissue culture plastic for at least 3 passages in standard ES cell medium containing LIF. Undifferentiated iPS C5 cells were then gently dissociated and plated onto $0.1 \%$ gelatin-coated tissue culture plastic at a density of $0.5 \times 10^{4}-1.5 \times 10^{4}$ cells $/ \mathrm{cm}^{2}$ in 1:1 mix of DMEM/F12 (Gibco) supplemented with $\mathrm{N} 2$ (Gibco) and Neurobasal medium (Gibco) supplemented with B27 (Gibco). To improve the efficiency of neural conversion, $1 \mu \mathrm{mol} \mathrm{L}{ }^{-1}$ all-trans retinoic acid (RA) (Sigma) was added to N2B27 medium in the initial $2 \mathrm{~d}$ of cell culture. Each medium was refreshed every other day.

\subsection{Immunocytochemistry}

Cells were fixed with $4 \%$ paraformaldehyde for $10 \mathrm{~min}$, and then blocked and permeabilized with 10\% FBS and $0.2 \%$ Triton X-100 for $30 \mathrm{~min}$ at room temperature. Primary antibodies were then applied and incubated at $4{ }^{\circ} \mathrm{C}$ overnight. Secondary antibodies anti-mouse Alexa Fluor 488/594 (Invitrogen) were applied to cells for $1 \mathrm{~h}$ at a dilution of $1: 2000$. Cell nuclei were stained with $1 \mu \mathrm{g} / \mathrm{mL} 4$, 6-diami- dino-2-phenylindole (Sigma). Then the cells were mounted with glycerine, and visualized and captured under a confocal microscope (Leica). The following primary antibodies were used in this work: mouse anti-Oct4 $(1: 100$, Santa Cruz Biotechnology), rabbit anti-Pax6 (1:200, Abcam), mouse anti-Nestin $(1: 200$, Abcam), mouse anti-Tuj1 (1:500, Abcam), mouse anti-MAP2 (1:200, Chemicon, Millipore), mouse anti-TH (1:500, Abcam), goat anti-Hb9 (1:200, Santa Cruz Biotechnology), mouse anti-GFAP (1:200, Cell Signaling Technology), and mouse anti-O4 (1:50, Stem Cell).

\subsection{Real-time PCR analysis}

Total RNA was isolated using Total RNA Isolation kit (Macherey-Magel). The first strand cDNA was synthesized with $0.2 \mathrm{mg}$ total RNA in $60 \mu \mathrm{L}$ using reverse transcriptase (TaKaRa) and olig-dgT primer. Real-time PCR analysis was performed on a MJR Chromo4 fluorescence quantitative PCR instrument (Bio-Rad) using the SYBR Green Premix Ex Taq (TaKaRa). The expression level of each gene at every checkpoint was normalized to the level of the housekeeping gene glyceraldehyde-3-phosphate dehydrogenase $(G A P D H)$.

\section{Results}

\subsection{Morphologic changes during neural induction}

The iPS C5 cells were subjected to a multistep protocol for differentiation into neural cells (Figure 1). Briefly, iPS C5 cells were plated at a low density and exposed to RA at a concentration of $1 \mu \mathrm{mol} \mathrm{L}{ }^{-1}$ for the initial $2 \mathrm{~d}$. After that cells were transferred into N2B27 medium without any exogenous growth factors. Undifferentiated iPS C5 cells formed colonies with a unique morphology when cultured on MEFs (mouse embryonic fibroblasts culture) (Figure 1(a)). Upon plating and culturing in neural differentiation medium, iPS C5 cells gradually adopted neural morphology by creating protrusions (Figure 1(d)).

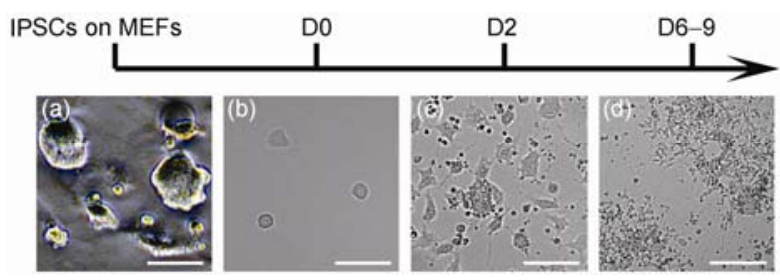

Figure 1 Schematic representation illustrating the sequential steps in the neural differentiation of iPS C5 cells. (a) iPS C5 cells were generated from mouse meningeal membranes and maintained on MEFs. (b) Subsequently, iPS C5 cells were replated and cultured on gelatin-coated plates. (c) iPS C5 cells were exposed to RA at concentrations of $1 \mu \mathrm{mol} \mathrm{L} \mathrm{L}^{-1}$ in the initial $2 \mathrm{~d}$. (d) After 6-9 d culture iPS C5 cells gradually create protrusions. Scale bar $=50 \mu \mathrm{m}$. 


\subsection{Expression of neural-specific genes in iPS C5 cells-derived neural cells}

To observe the process of iPS C5 cells differentiation, realtime PCR was used to evaluate the expression of neuroectodermal differentiation markers Sox1, Sox3 and Pax6, common neural progenitor marker Nestin, and early neuronal marker Tuj1. Both of these neural markers were up-regulated (D1-D6) during monolayer differentiation (Figure 2(b)). In contrast, Oct4 (also known as PouffI) and Nanog, two essential markers for undifferentiated ES cells were down-regulated in a time-dependent manner (Figure 2(a)). These findings confirmed that pluripotent meningealderived iPS cells were able to convert into neuropotent cells.

\subsection{Derivation of neural progenitor cells from mouse meningeal-iPS cells}

Immunocytochemistry analysis was used to investigate neural differentiation of iPS C5 cells in more detail. Sequential images showed time-dependent down-regulation of Oct4, which was consistent with real-time PCR results (Figures 2(a) and 3). After $6 \mathrm{~d}$ culture, most cells were immunonegative for Oct4 (Figure 3). In contrast, cells were immunopositive for Nestin and Pax6 and showed rosette conformations typical of neural progenitor properties at day 6 (Figure 4).

\subsection{Mouse meningeal-iPS cells generate neurons, astrocytes and oligodendrocytes}

Neural progenitor cells are multipotent and can give rise to three primary lineages of the CNS - neurons, oligodendrocytes and astrocytes [22]. In our protocol, the neuronal precursors began to express the neuronal markers Tuj1 and MAP2 on day 3 (not shown), and most cells showed positive staining for both markers on day 9 (Figures 5(a) and 6(a)). Interestingly, TH neurons and the motoneuron marker $\mathrm{Hb} 9$ also emerged during this period (Figures $5(\mathrm{~b})$ and 6(b)). However, few astrocytic marker GFAP-positive and mature oligodendrocytic marker O4-positive cells were observed after more than $9 \mathrm{~d}$ of differentiation in culture (Figure 7).

\section{Discussion}

Mouse meningeal-iPS C5 cells were successfully converted into neural stem cells using a revised protocol based on that described by Ying et al. [23] for neural differentiation of mouse ES cells. iPS C5 cells can form neural progenitors, and further differentiate into neuronal, astrocytic, and oligodendrocytic cells, showing that mouse meningeal-iPS cells have a potential comparable to that of ES cells.

Takahashi and Yamanaka [7] demonstrated direct reprogramming of somatic cells into a pluripotent state in 2006. More recently iPS cells were shown to be able to differentiate into many different cell types, such as hematopoietic, osteogenic cells, cardiac cells, adipogenic cells, and endothelial cells. Also, some iPS cell-derived somatic cells have already been used in disease models and, in some cases, have yielded functional improvement [7,19,24-28]. Recently, iPS cells have been generated through the use of different tissues, transcription factor combinations and selection methods. Considering all these different techniques, the differentiation ability of iPS cells should be thoroughly explored before they are used for in-depth studies [8-19]. Qin et al. [13] showed that cells from mouse meningeal membranes are highly amenable to generation of iPS because of the high expression of the embryonic master regulator Sox2. Meningeal-iPS C5 cells express high levels of endogenous ES markers, including Nanog, Oct4 (Figures 2(a) and (3)) and show highly homogeneous and complete reprogramming abilities enabling them to be used to produce chimeras [13]. However, whether meningeal-derived iPS cells can be converted into neural cells is still unknown.

Several reports have shown that iPS cells can be efficiently differentiated into neural types in vitro. Wernig et al. [29] have shown that iPS cells generated from mouse fibroblasts using 4 transcription factors Oct4, Sox2, Klf4, and
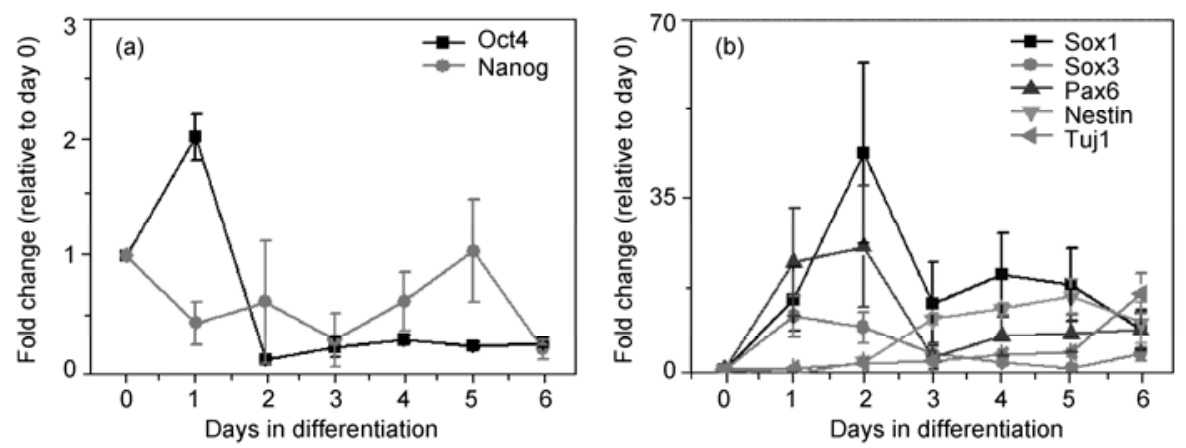

Figure 2 Gene expression analysis by real time-PCR on iPS C5 cells during neural differentiation. (a) The pluripotency markers Oct4 and Nanog were down-regulated; (b) conversely, the typical markers of neural progenitors Sox1, Sox3, Pax6 and Nestin were up-regulated. 
Oct4
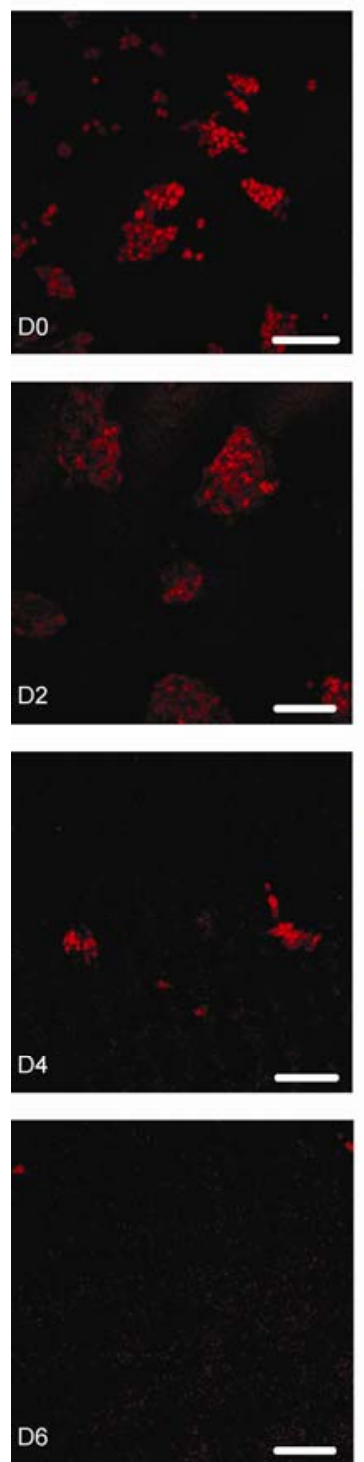

DAPI
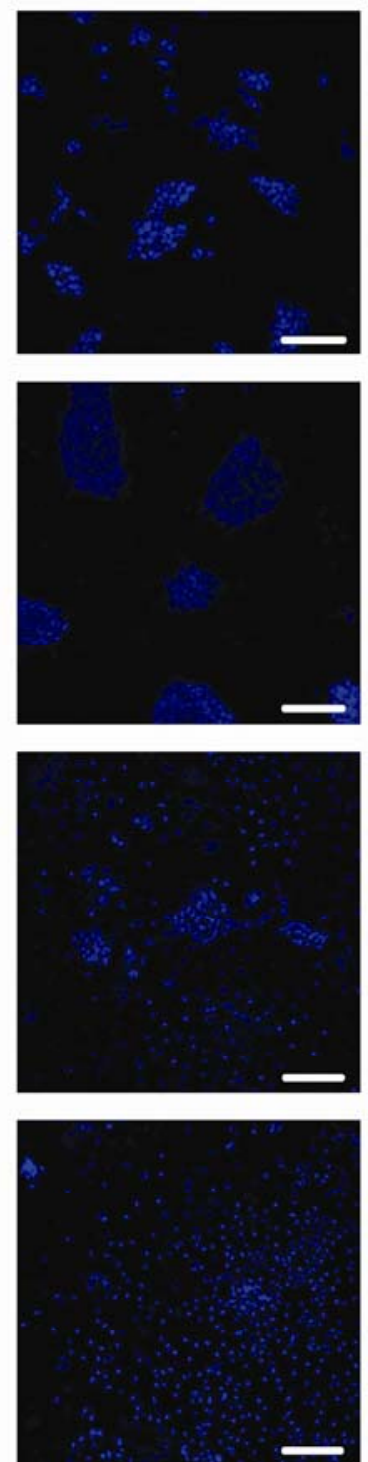

Merge
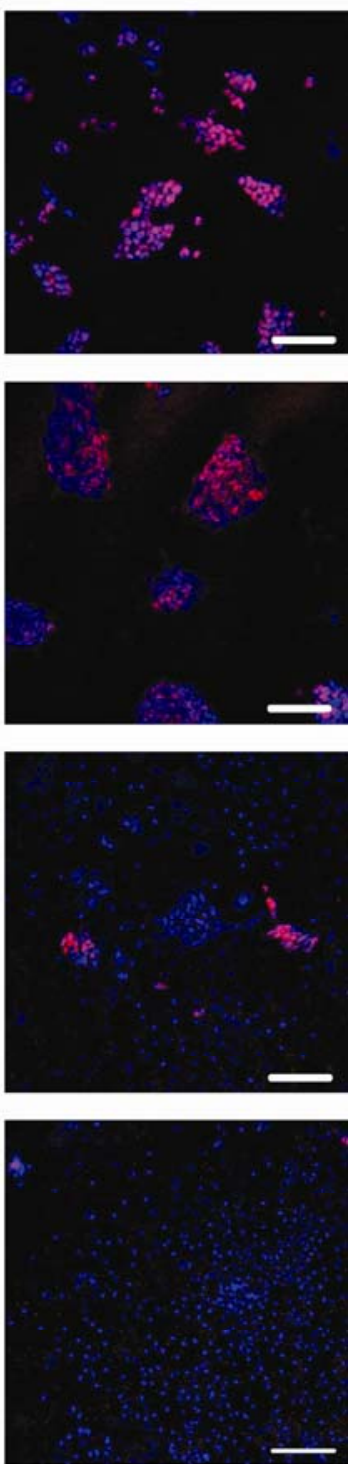

Figure 3 Immunofluorescence analysis showed that expression of Oct4 was down-regulated in a time-dependent manner and was undetectable at day 6. Scale bar $=100 \mu \mathrm{m}$.
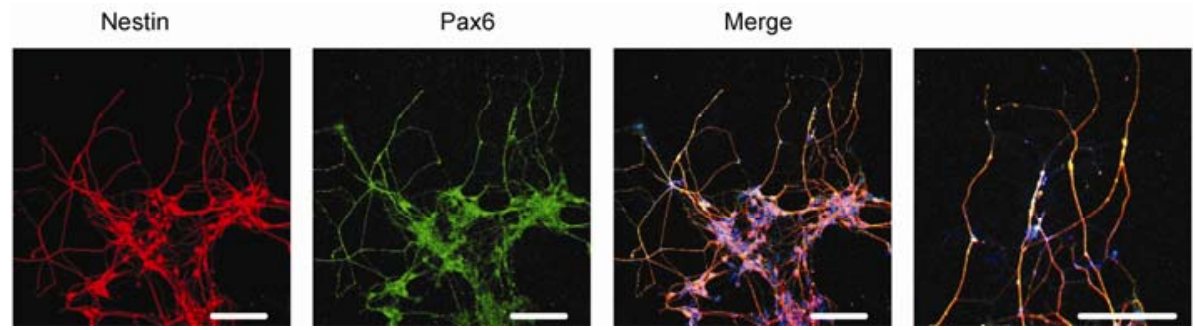

Figure 4 Immunofluorescence analysis showed that iPSC C5 cells were positive for markers of neural progenitors Pax6 and Nestin at day 6. Arrowhead denotes the formation of neural tube-like rosette structure. Scale bar $=100 \mu \mathrm{m}$.

c-Myc can be efficiently differentiated into neural precursor cells, giving rise to neuronal and glial cell types in vitro and functionally integrating into the fetal brain. Miura et al. [10] indicate that mouse iPS derived from MEF, TTF and hepa- tocyte cells can be induced to differentiate into neurons, astrocytes and oligodendrocytes both in vitro and in vivo. Different human iPS clones also share the same developmental time course as hESCs in response to the same neural 

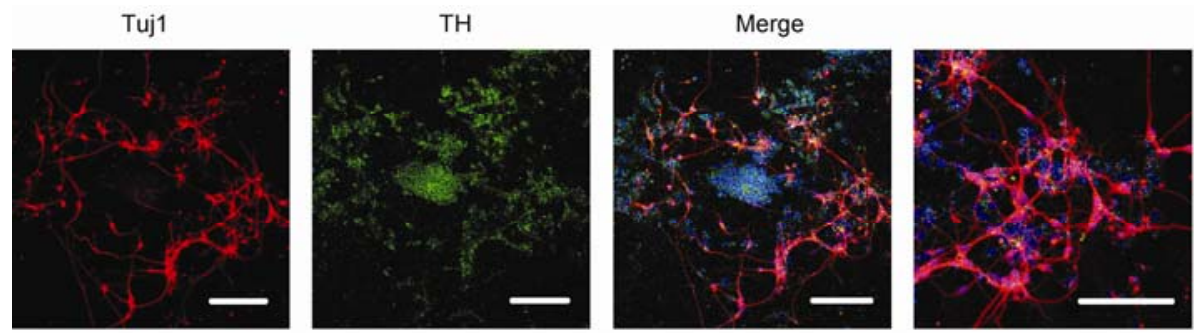

Figure 5 After iPSC C5 cells were cultured a in neural differentiation medium for 8-9 d, Tuj1 positive neurons and TH neurons were seen. Scale bar = $100 \mu \mathrm{m}$.

MAP2

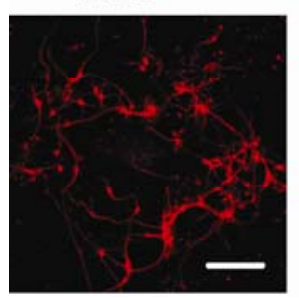

$\mathrm{Hb} 9$

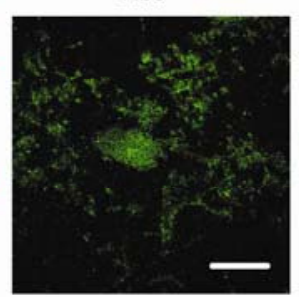

Merge

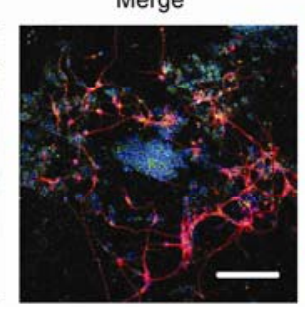

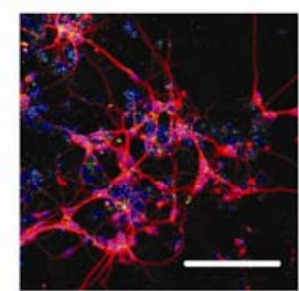

Figure 6 After iPSC C5 cells were cultured a in neural differentiation medium for 8-9 d, MAP2 positive neurons and Hb6 positive motor neurons emerged. Scale bar $=100 \mu \mathrm{m}$.
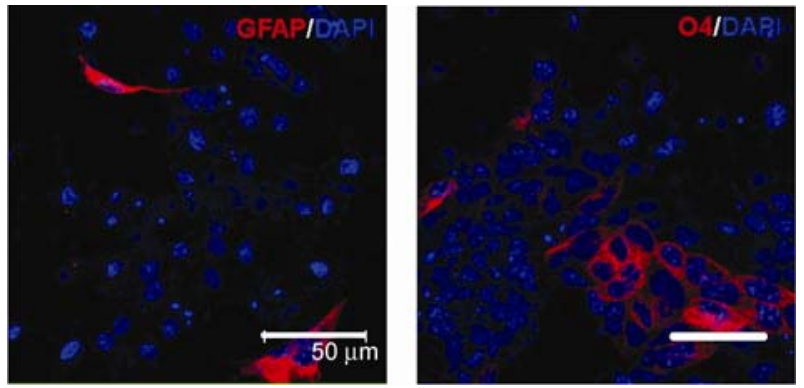

Figure 7 GFAP positive astrocytes (a) and O4 positive oligodendrocytes (b) can be detected at day 9 . Scale bar $=50 \mu \mathrm{m}$.

induction protocol [30]. Through our strategy, iPS C5 cells derived from meningeal membranes can be induced to express the neural markers Sox1, Sox3, Pax6, Nestin and Tuj1 in a time-dependent manner, while the ES marker Oct4 was promptly silenced during neural differentiation culture. This demonstrates that iPS cells are sensitive to the genetic switch that characterizes the change in potency of neural cells (Figure 2). This conclusion is also supported by the immunostaining results of neural markers Pax6 and Nestin and the ES marker Oct4 (Figures 3 and 4). Furthermore, like ES cells, neural progenitor cells generated from iPS C5 cells are multipotent and can give rise to 3 primary lineages of the CNS when detected by neuron markers Tuj1 and MAP2, astrocytic marker GFAP and oligodendrocytic marker O4 (Figures 5-7). It is interesting that neurons generated from iPS C5 cells are almost always tyrosine hydroxylase (TH) positive (Figure 4(b)). Previous studies showed that meningeal cells can induce differentiation of dopaminergic neurons from mouse and human ES cells [31]. Polo et al. [32] showed that iPSCs retain a transcriptional memory of their somatic cell of origin. Taking these views into account, we consider that $\mathrm{TH}$ positive cells generated from iPS C5 cells are associated with iPS C5 cells which retain some memory of meningeal cells. In our results, the expression level of Nanog was not decreased linearly (Figure 2(a)). Miura et al. [10] showed that secondary neurospheres derived from MEF-iPS cells contained fewer Nanog-EGFP + cells than those derived from TTF-iPS cells and hepatocyte-iPS cells. It seems that the persistent expression of pluripotent markers of iPS cell-derived somatic cells was associated with different tissues from which iPS cells originate. However, we think that it may have been caused by lentiviruses or retroviruses showing residual transgene expression during early-passage of iPS cells, because different iPS clones generated using the same tissues and transcription factor combinations also showed different neural differentiation efficiency [30].

Previous reports claim that mouse neural stem cells and meningeal membranes both express high levels of Sox2 and are amenable to induction into iPS [13,20,21]. However, meningeal-derived iPS are more suitable for cell transplantation therapies because of the source limitation of neural stem cells. Our work shows that iPS C5 cells share the neural differentiation ability of ES cells. Finally, the in vitro neural differentiation of iPS cells provides a new perspective for studying the cellular and molecular mechanisms of early neural system development and generation of patientspecific cells for therapeutic applications in dysfunctional or aging neural tissues. 
We would like to thank Dr. PEI DuanQing for kindly providing us with mouse iPS C5 cells. This work was supported by the National Basic Research Program of China (2007CB947804), the Joint Funds of the National Natural Science Foundation of China-Guangdong Province (U0972001/L02), the National Natural Science Foundation of China (30700213/C090204) and the Natural Science Foundation of Guangdong Province (07007215).

1 Wichterle H, Przedborski S. What can pluripotent stem cells teach us about neurodegenerative diseases? Nat Neurosci, 2010, 13: 800-804

2 Przedborski S, Vila M, Jackson-Lewis V. Neurodegeneration: What is it and where are we? J Clin Invest, 2003, 111: 3-10

3 Bjorklund A, Lindvall O. Cell replacement therapies for central nervous system disorders. Nat Neurosci, 2000, 3: 537-544

4 Goldman S. Stem and progenitor cell-based therapy of the human central nervous system. Nat Biotechnol, 2005, 23: 862-871

5 Lindvall O, Kokaia Z. Stem cells for the treatment of neurological disorders. Nature, 2006, 441: 1094-1096

6 Rossi F, Cattaneo E. Opinion: Neural stem cell therapy for neurological diseases: Dreams and reality. Nat Rev Neurosci, 2002, 3: 401-409

7 Takahashi K, Yamanaka S. Induction of pluripotent stem cells from mouse embryonic and adult fibroblast cultures by defined factors. Cell, 2006, 126: 663-676

8 Tat P A, Sumer H, Jones K L, et al. The efficient generation of induced pluripotent stem (iPS) cells from adult mouse adipose tissue-derived and neural stem cells. Cell Transplant, 2010, 19: 525-536

9 Giorgetti A, Montserrat N, Rodriguez-Piza I, et al. Generation of induced pluripotent stem cells from human cord blood cells with only two factors: Oct4 and Sox2. Nat Protoc, 2010, 5: 811-820

10 Miura K, Okada Y, Aoi T, et al. Variation in the safety of induced pluripotent stem cell lines. Nat Biotechnol, 2009, 27: 743-745

11 Haase A, Olmer R, Wunderlich S, et al. Generation of induced pluripotent stem cells from human cord blood. Cell Sten Cell, 2009, 5: 434-411

12 Stadtfeld M, Nagaya M, Utikal J, et al. Induced pluripotent stem cells generated without viral integration. Science, 2008, 322: 945-949

13 Qin D J, Gan Y, Shao K F, et al. Mouse meningiocytes express Sox2 and yield high efficiency of chimeras after nuclear reprogramming with exogenous factors. J Biol Chem, 2008, 283: 33730-33735

14 Nakagawa M, Koyanagi M, Tanabe K, et al. Generation of induced pluripotent stem cells without Myc from mouse and human fibroblasts. Nat Biotechnol, 2008, 26: 101-106

15 Kim J B, Zaehres H, Wu G, et al. Pluripotent stem cells induced from adult neural stem cells by reprogramming with two factors. Nature, 2008, 454: 646-650

16 Eminli S, Utikal J, Arnold K, et al. Reprogramming of neural progenitor cells into induced pluripotent stem cells in the absence of exogenous Sox2 expression. Stem Cells, 2008, 26: 2467-2474

17 Aoi T, Yae K, Nakagawa M, et al. Generation of pluripotent stem cells from adult mouse liver and stomach cells. Science, 2008, 321: 699-702

18 Aasen T, Raya A, Barrero M J, et al. Efficient and rapid generation of induced pluripotent stem cells from human keratinocytes. Nat Biotechnol, 2008, 26: 1276-1284

19 Park I H, Arora N, Huo H, et al. Disease-specific induced pluripotent stem cells. Cell, 2008, 134: 877-886

20 Kim J B, Sebastiano V, Wu G M, et al. Oct4-induced pluripotency in adult neural stem cells. Cell, 2009, 136: 411-419

21 Kim J B, Zaehres H, Wu G M, et al. Pluripotent stem cells induced from adult neural stem cells by reprogramming with two factors. Nature, 2008, 454: 646-650

22 Conti L, Pollard S M, Gorba T, et al. Niche-independent symmetrical self-renewal of a mammalian tissue stem cell. PLoS Biol, 2005, 3: e283

23 Ying Q L, Stavridis M, Griffiths D, et al. Conversion of embryonic stem cells into neuroectodermal precursors in adherent monoculture. Nat Biotechnol, 2003, 21: 183-186

24 Dimos J T, Rodolfa K T, Niakan K K, et al. Induced pluripotent stem cells generated from patients with ALS can be differentiated into motor neurons. Science, 2008, 321: 1218-1221

25 Hanna J, Wernig M, Markoulaki S, et al. Treatment of sickle cell anemia mouse model with iPS cells generated from autologous skin. Science, 2007, 318: 1920-1923

26 Hirami Y, Osakada F, Takahashi K, et al. Generation of retinal cells from mouse and human induced pluripotent stem cells. Neurosci Lett, 2009, 458: 126-131

27 Gai H, Leung E L, Costantino P D, et al. Generation and characterization of functional cardiomyocytes using induced pluripotent stem cells derived from human fibroblasts. Cell Biol Int, 2009, 33: 11841193

28 Taura D, Noguchi M, Sone M, et al. Adipogenic differentiation of human induced pluripotent stem cells: Comparison with that of human embryonic stem cells. FEBS Lett, 2009, 583: 1029-1033

29 Wernig M, Zhao J P, Pruszak J, et al. Neurons derived from reprogrammed fibroblasts functionally integrate into the fetal brain and improve symptoms of rats with Parkinson's disease. Proc Natl Acad Sci USA, 2008, 105: 5856-5861

30 Hu B Y, Weick J P, Yu J Y, et al. Neural differentiation of human induced pluripotent stem cells follows developmental principles but with variable potency. Proc Natl Acad Sci USA, 2010, 107: 4335-4340

31 Hayashi H, Morizane A, Koyanagi M, et al. Meningeal cells induce dopaminergic neurons from embryonic stem cells. Eur J Neurosci, 2008, 27: 261-268

32 Polo J M, Liu S, Figueroa M E, et al. Cell type of origin influences the molecular and functional properties of mouse induced pluripotent stem cells. Nat Biotechnol, 2010, 28: 848-855

Open Access This article is distributed under the terms of the Creative Commons Attribution License which permits any use, distribution, and reproduction in any medium, provided the original author(s) and source are credited. 